\title{
Effectiveness of Remote Ischaemic Conditioning in Critical Ischemia of the Digitals of Upper and Lower Limbs
}

Nadjib Schahab ${ }^{1 \# *}$, Adem Aksoy ${ }^{1 \#, ~ S c h a m i m ~ S c h a h a b ², ~ M a r t i n ~ S t e i n m e t z ~}{ }^{1}$, Christian Berg ${ }^{2}$, Christian Schaefer ${ }^{1}$, Georg Nickenig ${ }^{1}$ and Vedat Tiyerili ${ }^{1}$

${ }^{1}$ Department of Cardiology, Angiology, Pneumology, University Hospital Bonn, Germany

${ }^{2}$ Department of Angiology, Evangelisches Krankenhaus Mettmann, Germany

\#The authors contributed equally

\begin{abstract}
Aim: The aim of our study was to determine the effectiveness and safety of remote ischaemic conditioning (RIC) in combination with i.v. prostavasin as a method of treatment for patients with peripheral acral vasculopathy, presenting as critical ischemia of the digitals of upper and lower limbs at the time of presentation with either surgical nor interventional treatment option
\end{abstract}

Method: 33 patients were included in this non-randomized, prospective pilot-study with compassionate use. Primary endpoints were healing of ulcerations and amputation free time, secondary endpoint was safety of RIC.

For the RIC, a blood flow reduction in the extremities was held in 2 cycles for over 5 minutes $(20 \mathrm{mmHg}$ over the systolic blood pressure) with 5 minutes reperfusion. During this time the medical agents were infused through a superficial vein into index extremity transvenously. The medication included: 2500 i.e., Heparin bolus, $5 \mu$ g PGE-1 in a $50 \mathrm{~mL}$ syringe with an isotonic solution. In cases where a vascular occlusion was seen in angiography additionally to PGE-1, 20 mg rt-PA was infused.

Results: In 33 cases, transcutaneous oxygen partial pressure $\left(\mathrm{TcPO}_{2}\right)$ levels increased significantly from $33 \pm 16$ $\mathrm{mmHg}$ to $48 \pm 13 \mathrm{mmHg}(p=0.0005)$ after the therapy compared to baseline. In 33 cases there was an improvement from $55 \pm 27 \mathrm{mmHg}$ to $73 \pm 27 \mathrm{mmHg}(p \leq 0.004)$ in the acral arterial pressure measurement. 31 patients showed significantly better perfusion and vascularisation of the ischemic extremity after the therapy demonstrated in acral oscillography $(31 / 94 \%)$ and angiography $(6 / 18 \%)$. In 16 cases ulcerations $(n=20)$ healed after 15 sessions of RIC. There was no need of any major or minor amputation in all patients.

Conclusion: The presented RIC therapy proved to be an effective and safe treatment in addition to best medical treatment for patients with acute acral vascular disorder, especially in acute digital ischaemia, without a surgical or an interventional option.

Keywords: Remote ischeamic conditioning; Peripheral acral vascular disease; $\mathrm{TcPO}_{2}$; Acral arterial pressure; Prostavasin

\section{Introduction}

The pathophysiology of acral vasculopathy is a complex and in most cases chronic process affecting the macrovascular and microvascular circulation of muscle and non-muscle tissues with or without trophic skin changes or tissue loss. The terminal stream bed is essential for cell nutrition, regulation of temperature and clearance of the skin. Temperature regulation is done by the shunts (arterio-venous anastomoses). Acral vasculopathies occurs as ulcerations, vascular occlusions, malformations, vascular neoplesia, and metabolic debris or even as vasculitis.

Secondary vasculopathy appears as consequence of systemic diseases, primarily rheumatic diseases and infections. Secondary systemic vasculitis can be a consequence of collagenosis like systemic sclerosis or rheumatoid arthritis $[1,2]$. Especially in diabetes mellitus the hyperglycemia has multiple consequences on cell structure and blood flow [3]. Special importance has to be assigned to the endothelium of the blood vessels. Destruction of the cellular structure of the endothelium leads to changes in the balance of relaxing (NO, Prostacyclin) and constrictive factors (Angiotensin II, Prostaglandin $\mathrm{H} 2$ ), resulting in so called endothelial dysfunction [3]. Endothelial dysfunction, reduced vasodilation and decreased perfusion, can cause ischemia and ulcerations of fingertips and toes [4]. Finally, hypoxia supports bacterial growth and infections, with the consequence of impaired healing potential in ulcer leading to limb amputation [5] For such chronic injuries, pharmaceuticals like antiplatelet agents or intravenous prostanoids, Prostaglandin E1 (PGE-1, Prostavasin ${ }^{\circledR}$ ), rheological agents, i.v. heparin and in case of vascular occlusion systemic i.v. recombinant tissue plasminogen activator (rtpa) are firstline approach $[2,6,7]$. Surgical treatment of limb injuries consists of digital sympathectomy, arterial reconstruction and amputation [8]. Bier published in 1908 [9] first a therapeutic strategy for anesthesia purpose: over the complete arterial and venous stasis of the upper or lower limb (so called Bier's block) it is possible to fill the empty venous system with the acquired amount of medication. The basic idea behind the Bier block is to exsanguinate the extremity, apply an arterial tourniquet to isolate it from circulation, and inject local anesthetic into the extremities venous system, inducing anesthesia. Murry et al. brought Ischemic

*Corresponding author: Nadjib Schahab, Department of Cardiology, Angiology and Pneumology, University Hospital Bonn, Germany, Tel: 004922828716670; E-mail: nadjib.schahab@ukb.uni-bonn.de

Received May 29, 2017; Accepted June 26, 2017; Published June 29, 2017

Citation: Schahab N, Aksoy A, Schahab S, Steinmetz M, Berg C, et al. (2017) Effectiveness of Remote Ischaemic Conditioning in Critical Ischemia of the Digitals of Upper and Lower Limbs. J Vasc Med Surg 5: 321. doi: 10.4172/23296925.1000321

Copyright: (c) 2017 Schahab N, et al. This is an open-access article distributed under the terms of the Creative Commons Attribution License, which permits unrestricted use, distribution, and reproduction in any medium, provided the original author and source are credited. 
preconditioning into notice in 1986 with same strategy as a therapeutic strategy in order to alleviate ischemia-reperfusion-induced injury [10]. In remote ischemic conditioning (RIC), brief, reversible episodes of ischemia with reperfusion in one vascular bed, tissue or organ confer a global protective phenotype and render remote tissues and organs resistant to ischemia/reperfusion injury. The peripheral stimulus can be chemical, mechanical or electrical and involves activation of peripheral sensory nerves. Solid evidence from experimental and clinical studies supports protection by RIC from ischemia/reperfusion (I/R) injury of the heart and other organs [11]. Given its advantage of avoiding invasive procedures, the RIC technique using transient limb ischaemia as a stimulus has emerged as an attractive strategy in various clinical settings. RIC was proven to be able to attenuate I/R injury in patients undergoing cardiac surgery, elective surgery for abdominal aortic aneurysm, elective percutaneous coronary intervention and reperfusion in acute myocardial infarction [12-14]. However, the potential clinical benefit of any of these findings remains to be seen and to our best knowledge, the RIC technique has not been used in treatment of peripheral vasculopathy or ischemic lesions.

Ferreira [15] used and modified this method in the late 80s and 90s, calling it retrograde venous perfusion (RVP). Since then, readers find the terms RVP and RTVP in literature. Giving the drugs transvenously it is a possibility to break the capillary diffusion barrier and to deliver medication retrograde to the peripheral arterial system. After application of a vessel barrier like an inflatable cuff and infusion of medicals the pharmaceuticals reach the microcirculation by retrograde diffusion. High pressure enlarges the capillary surface and high agent concentration promotes the diffusion gradient. Therefore, we hypothesised, that it is quite possible that use of this strategy may help to increase blood flow and microcirculation, especially when drugs are administered locally and transvenously during RIC episode. The aim in this study was to determine the effectiveness and safety of the RIC combined with retrograde transvenous perfusion therapy [9], as a method of treatment in patients presenting with critical. Because all cases were acute, immediate intervention was indicated. The aim was a local therapy. We wanted to show the efficacy of RIC in cases of disturbances of microcirculation to avoid further ulcerations, necrosis and at least amputations of the extremities. In the presented study, the medication was assigned to be infused by retrograde transvenous perfusion in the index extremity under the assumption, that the duration of medical contribution increases at the location of the lesion and thus can take full effect.

\section{Materials and Methods}

In this study, RIC was applied in 33 patients. It was a non-randomized study because each patient with a lesion not treatable with surgical or interventional therapy and best medical treatment got this treatment after explanation and agreement. Presentation to the ethic commission was not requested because this study dealt with compassionate use and there was no use of any investigational drug. All medical agents given in this study can be used intravenously. The intravenous regional route was chosen as an alternative and effective route of administration at the location of the lesion. We gave clarification and got patient's written informed consent. Primary endpoints were healing of ulceration and amputation free time, secondary endpoint was safety of RIC. All 33 women and men were patients with acral lesions in the Department of Angiology of the University Hospital Essen and Bonn taken in for further treatments. The collective consisted of 21 males and 12 females, aged between 20 and 84 years, average of $53 \pm 15$ years. In total 33 extremities (20 upper extremity and 13 lower extremity) were treated. All patients presented acral vascular occlusions associated with pain, blue toes and fingers with existing ulceration $(n=20)$ or risk of ulceration. In 18 cases it was based on embolic/thrombotic peripheral vessel occlusion and in 15 cases based on progressed secondary Raynaud syndrome with vasospastic component. Patients with typically primary Raynaud attacks were excluded. 6(18\%) patients had a diabetes mellitus, $6(18 \%)$ coronary artery disease. There were $14(42 \%)$ patients with diagnosed collagenosis (Sharp, Scleroderma, Fibromylagia, CREST) (Table 1). Clinically, RIC stimulus was generally delivered by inflating the blood pressure cuff tied on the index extremity $20 \mathrm{mmHg}$ higher than the systolic blood pressure, rendering the extremity ischemic for $5 \mathrm{~min}$, followed $5 \mathrm{~min}$ reperfusion by deflating the cuff. This cycle was repeated for 2 consecutive periods to precondition the tissue and improve the perfusion. During this time with artificially discontinued arterial circulation the medical agents were infused through a superficial vein of index extremity within 10 minutes according to predetermined regimes. The medication included: 2500 i.e., Heparin bolus, $5 \mu \mathrm{g}$ PGE1 in a $50 \mathrm{~mL}$ syringe with an isotonic solution and in 18 cases with angiographically diagnosed vessel occlusion in addition 20 mg rt-PA (Figure 1). In average all patients had 14 sessions $(14+/-5$ days on a daily basis). In addition to the mentioned agents all patients received PGE-1 $40 \mu \mathrm{g}$ (in 250ml NACL 0.9\%) twice a day over 4 hours and Heparin i.v. in effective dose. In all patients underwent daily RIC several basic and dynamic macro- and microcirculation functions were controlled before and after treatment. We measured acral pulse oscillography, acral blood pressure measurement, angiography $(n=22)$ and microcirculation function by transcutaneous oxygen partial pressure $\left(\mathrm{TcPO}_{2}\right)$.

\section{Acral pulse oscillography}

Optical sensors measure the acral pulsation. Different sensor types can be selected: $\lambda$ transmission sensors $\lambda$ reflexion sensors

\begin{tabular}{|l|c|}
\hline N & 33 \\
\hline Male & $21(63 \%)$ \\
\hline Age, yrs & $53+1-15$ \\
\hline Hypertension & $17(51 \%)$ \\
\hline Diabetes mellitus & $6(18 \%)$ \\
\hline Ischemic heart disease & $6(18 \%)$ \\
\hline progressed secondary Raynaud syndrome & $15(45 \%)$ \\
\hline Collagenosis (Sharp, Sclerodermia, Fibromyalgia, CREST) & \\
\hline Current smoker & $27(82 \%)$ \\
\hline Upper limb & $20(60 \%)$ \\
\hline Lower extremity & $13(39 \%)$ \\
\hline Prostavasin (PGE) & $33(100 \%)$ \\
\hline Lysis & $18(55 \%)$ \\
\hline Vessel occlusion & $18(55 \%)$ \\
\hline
\end{tabular}

Table 1: Baseline charachteristics.

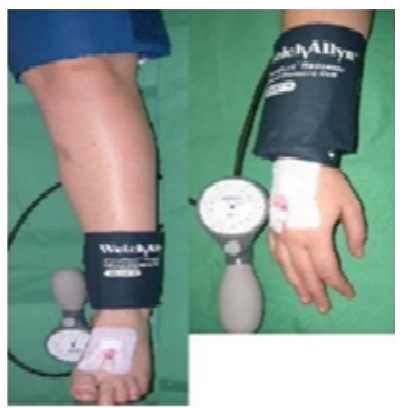

Figure 1: Agents were infused through a superficial vein during RIC. 
Citation: Schahab N, Aksoy A, Schahab S, Steinmetz M, Berg C, et al. (2017) Effectiveness of Remote Ischaemic Conditioning in Critical Ischemia of the Digitals of Upper and Lower Limbs. J Vasc Med Surg 5: 321. doi: 10.4172/2329-6925.1000321

Page 3 of 5

$\lambda$ microcirculation sensors. We used microcirculation sensors (Vasolab5000 Elcat).

\section{Acral blood pressure measurement}

Acral blood pressure measurement was carried out with congestion cuffs (Vasolab5000 Elcat). Optical sensors were used to record pulsation (acral pulse oscillography). The pneumatic system ensures a correct inflation of the cuffs and also controls the deflation.

\section{$\mathrm{TcPO}_{2}$}

The $\mathrm{TcPO}_{2}$ is a measurement of oxygen partial pressure $\left(\mathrm{PO}_{2}\right)$ taken from the skin, it is an indicator of working microcirculation.

$\mathrm{TcPO}_{2}$ was measured by oxymeter (TCM3 Radiometer Copenhagen, Denmark) at stable room temperature, the electrode temperature being $45^{\circ} \mathrm{C}$. The measurements were performed on patients in a supine position at rest, on index extremity and mostly near the level where ischemia had been anticipated clinically. After applying the electrodes to the skin with an adhesive ring, $\mathrm{TcPO}_{2}$ values were measured continuously and registered automatically for 15 to 20 minutes. The results were statistically analysed by IBM SPSS Statistics version 20 and Microsoft Exel 2010.

\section{Results}

\section{Oxygen measurement}

After the therapy $\mathrm{TcPO}_{2}$ levels increased significantly from $33 \pm$ 16 (means \pm standard deviation) $( \pm 2.78 \mathrm{SEM}=$ standard error of the mean) $\mathrm{mmHg}$ to $48 \pm 13( \pm 2.25 \mathrm{SEM}) \mathrm{mmHg}(\mathrm{p}=0.0005)$ compared to baseline (Figure 2 and Table 2).

\section{Acral oscilography and blood pressure}

In the acral arterial pressure measurement there was a remarkable improvement from $55 \pm 27$ ( \pm 4.73 SEM $) \mathrm{mmHg}$ to $73 \pm 27( \pm 4.76$ SEM) $\mathrm{mmHg}(\mathrm{p} \leq 0.004)$ (Figure 3 and Table 2). Shortly after the RIC in digital subtraction angiography (DSA) we saw especially in

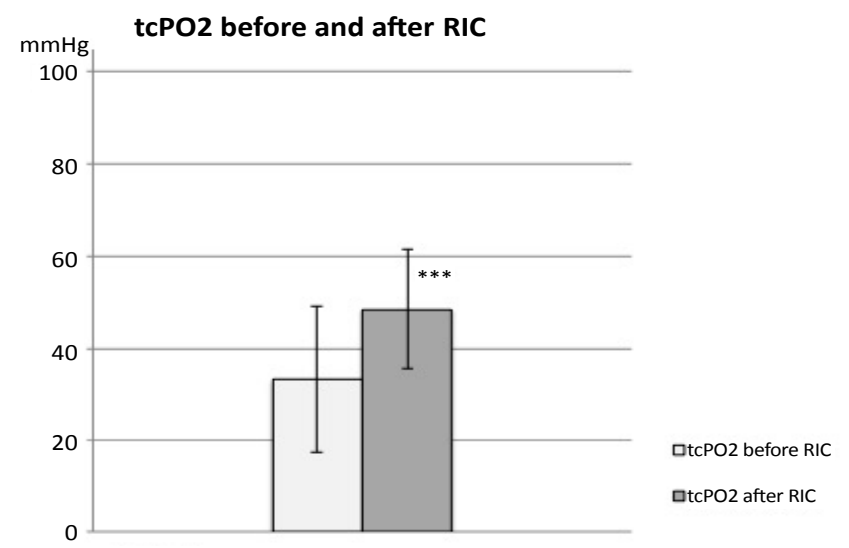

Figure 2: $\mathrm{TcPO}_{2}$ before and after RIC.

\begin{tabular}{|l|c|c|}
\hline tcPO2 before RIC & $33 \pm 16 \mathrm{mmHg}$ & $\mathrm{p}=0.0005$ \\
\hline tcPO2 after RIC & $48 \pm 13 \mathrm{mmHg}$ & \\
\hline $\begin{array}{l}\text { Acral Blood Pressure } \\
\text { Measurement before RIC }\end{array}$ & $55 \pm 27 \mathrm{mmHg}$ & $\mathrm{P} \leq 0.004$ \\
\hline $\begin{array}{l}\text { Acral Blood Pressure } \\
\text { Measurement after RIC }\end{array}$ & $73 \pm 27 \mathrm{mmHg}$ & \\
\hline
\end{tabular}

Table 2: $\mathrm{tcPO}_{2}$ and acral blood pressure measurement before and after RIC. the region of the lesion vasodilated vessels compared to the initial findings (Figure 4A). There was an increase of the total blood supply to the extremities immediately after a RIC session (Figures $4 \mathrm{~B}$ and $4 \mathrm{C}$ ). One week after the RIC angiographic diagnosis showed an appropriate vessel presentation with good visible collateral vessels (Figure 4C). In $16(80 \%)(n=20)$ cases ulcerations healed after 15 sessions (Figure 5). There was no need of any major or minor amputation in all patients. In 6 cases the therapy had to be paused for 3 days because of local haematoma and oedema, which were completely reversible.

\section{Discussion}

In most of cases peripheral acral vasculopathy presents as a typical Raynaud's phenomenon, ischemic pain at rest, ulcers or gangrene. Up to the present, to our knowledge there are no conclusive evidence based studies dealing with the treatment of patients with acral vasculopathy. Reasons here for are the wide variety of symptoms and

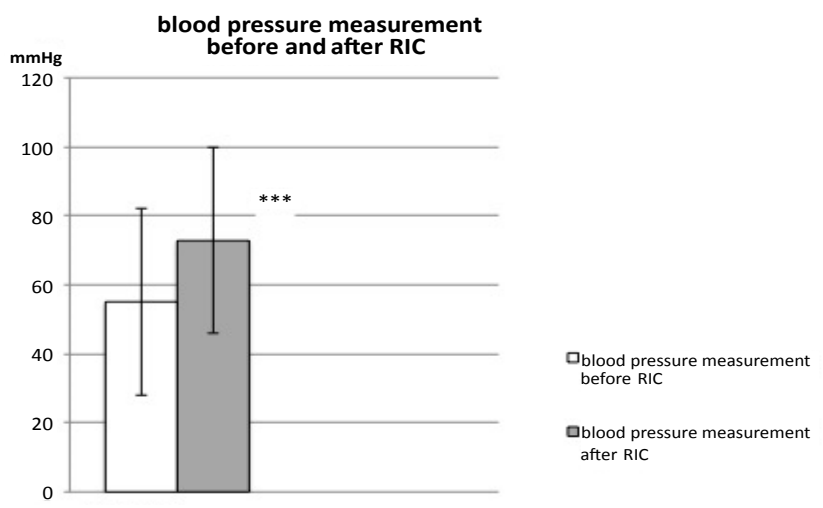

Figure 3: Acral arterial pressure measurement before and after RIC.
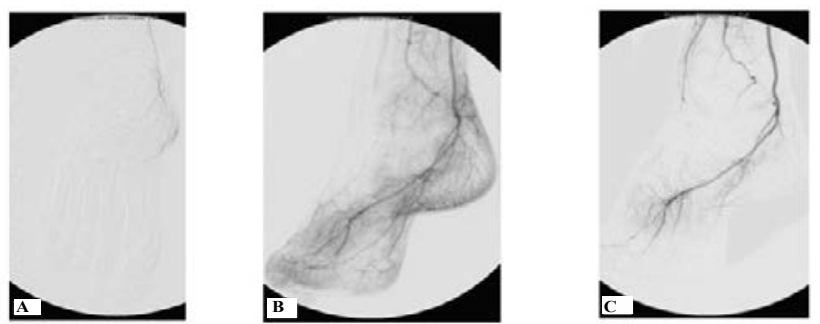

Figure 4: Digital subtraction angiography; (A) initial findings, (B) shortly after RIC and (C) one week after the RIC.
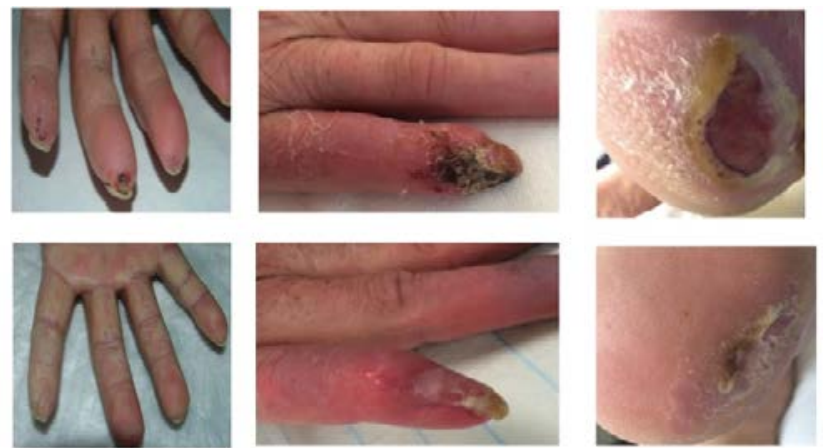

Figure 5: Ulcerations healed after RIC 
pathophysiological mechanisms involved, especially in secondary Raynaud's phenomenon. The aim of the presented study was to treat the afflictions symptomatically. The primary endpoint was complete healing without pain or major amputation, ulcer healing or at least reduction in ulcer area size. However, we could show that the method of RIC with RTVP is suitable for acute chronic ulcerations. We observed healing of the ulcerations in $80 \%$ and at least none of the patients had to have an amputation and the method is safe without any serious complications. Our findings are in coincidence with former studies. Agarwal et al. [16] treated nine diabetic and 10 ischemic non-healing pedal ulcers with RTVP and soda bicarbonate, heparin, lignocaine, gentamicin and pentoxiphylline. Five of the nine diabetic non-healing ulcers showed complete healing after 10-24 days (mean 16 days), while ischemic ulcers took 10-14 days for complete recovery (mean 13.6 days). All 10 patients with ischemic disease had a reduction of rest pain. Five of the ischemic patients showed complete healing of ulcers. In two patients, pre-gangrene changes were reversed. BühlerSinger et al. [17] treated 14 patients with diabetic neuropathic plantar ulcers (DNPU) at three differently affected sites (dorsal forefoot, ulcer edge, lower leg). After 10 days of RTVP a group of 7 patients showed remarkable improvement of laser-Doppler flux (LDF) and cutaneous oxygen tension $\left(\mathrm{pCUO}_{2}\right)$ on the forefoot and the lower leg. The authors suggest this as hint for better oxygen supply to the tissues after RTVP therapy. Partsch [18] used the RTVP in ten patients with large resistant leg ulcers by retrograde local pressure infusions of 100 000 IU of urokinase three times per week for a total of 3-11 weeks. The measurement of Doppler flowmetry showed clinical improvement in 9 patients. Kommissari [19] evaluated data of 20 patients with pedal lesions who had been treated by RTVP between 1998 and 2002. Data showed for all patients' stable function of the treated extremities.

\section{Remote ischemic conditioning}

Details of the mechanisms for local release of the protective signal at the remote site and the contributions of neuronal and humoral pathways are not yet clear, not only in signal release, but also in signal transfer to the target organ and protective signal transduction within the target organ. Repeated brief inflation/ deflation of a blood pressure cuff at the arm, leg, or both is easily feasible, noninvasive, inexpensive, effective, and safe $[13,14]$.

RIC is known to protect tissues against I/R injury that occur in several clinical settings such as cardiac surgery with cardiopulmonary bypass and ST-segment elevation myocardial infarction. However, the mechanism through which RIC exerts its protection is still unclear. RIC is believed to produce protective signals that are conveyed from the remote tissue to the target organ. Several mechanisms may be involved, including blood-borne factor release, neuronal pathway activation and systemic response participation. These protective signals may activate intracellular survival signaling pathways in the target organ. Several studies have reported the involvement of endogenous factors in protective mechanisms, such as adenosine, endocannabinoids, bradykinin, opioids, and erythropoietin [14]. The actual identity of circulating humeral factors currently remains, however, unknown.

Acute digital ischemia is a condition in which patients experience a decline in quality of life because of pain, ulceration related to limb ischaemia. On this basis, we hypothesized that RIC could improve perfusion capacity in patients with acute ischemia. Some studies have suggested that RIC might modify skeletal muscle tolerance to tissue hypoxia, thereby increasing maximal exercise performance. Furthermore, previous studies have demonstrated the potential beneficial effects of ischemic preconditioning through functional changes in the vasculature and adaptions of the skeletal muscles [12-14].

The mechanisms of action are still under speculation. Fibrinolytic effects contribute as well as decrease of blood viscosity. Restoring of endothelial function may also be a part of improvement [18]. The increase of venous pressure is a further effect. Langer et al. [20] found by ultrastructural studies that RTVP induced a dilatation of venous capillaries and postcapillary venules and a widening of the space between endothelial cells and pericytes. In our study the results of increased oxygen pressure (increased $\mathrm{TcPO}_{2}$ levels) give reason to suggest that better oxygen supply and transport contributes to healing.

Other angiologic studies give a hint that the block of the A. brachialis leads to a flow-mediated dilatation [21]. In the course of this vasodilatation the result is a better perfusion.

Further well-conducted, high quality randomised trials with high number of homogenous cases are needed to prove the efficiency of this therapy for example comparing RIC and systemic i.v. PGE-1 and systemic i.v. PGE-1 without RIC.

\section{Study limitation}

This study was not designed to determine the cause of vasculopathies. This study also did not evaluate the exact mechanism(s) by which RIC improved the microcirculation. It was a non-randomized prospective study with a heterogeneous group of clinical disorder with different risk factors, aetiology and stage of symptoms and small sample size.

We only tested one RIC protocol, exploring the early phase of conditioning. It is as yet unknown if other RIC sequences and other timings would have induced similar results. Finally, the limited sample size of the study may have underpowered the study to detect any change after RIC and the effects are likely to be overestimated. Therefore controlled trial with a double blinded design is to be carried out according to a randomization protocol.

\section{Conclusions}

The presented therapy with RIC by infusion of medication over RTVP proved to be an effective and safe treatment on top of best medical treatment for patients with acute acral vascular disorder. RIC can reach an improvement of microcirculatory parameters suggesting that a better oxygen supply to the tissue supports well perfusion and cell metabolism. The main impact of the presented study was to find a method to promote healing, preventing lesions and ulcerations and at least preventing amputations of the extremities.

\section{References}

1. Zeller T, Cissarek T, Gray WA, Kröger K (2009) Gefäßmedizin: Therapy and practice. ABW Science Publishing Company, Berlin.

2. Bakst R, Merola JF, Franks AG, Sanchez M (2008) Raynaud's phenomenon pathogenesis and management. J Am Acad Dermatol 59: 633-653.

3. Siegenthaler W, Blum HE (2006) Klinische Pathophysiologie. Georg Thieme Verlag Stuttgart, Auflage.

4. Gary T, Brodmann M (2007) Raynaudphänomen. Dfp rheuma 4:17-20.

5. Harrison DH (2003) Optical measurement of tissue oxygen saturation in lower limb wound healing. Adv Exp Med Biol 540: 265-269.

6. Drinda S, Neumann T, Pöhlmann G, Vogelsang H, Stein G, et al. (2005) The response of skin perfusion and of rheological immunological variables to intravenous prostanoid administration in Raynaud's phenomenon second collagenosis. Vasa 34: 243-249.

7. McMahan ZH, Wigley FM (2010) Raynaud's phenomenon and digital ischemia 
Citation: Schahab N, Aksoy A, Schahab S, Steinmetz M, Berg C, et al. (2017) Effectiveness of Remote Ischaemic Conditioning in Critical Ischemia of the Digitals of Upper and Lower Limbs. J Vasc Med Surg 5: 321. doi: 10.4172/2329-6925.1000321

Page 5 of 5

a practical approach to risk stratification diagnosis and management. International Journal of Clinical Rheumatology 5: 355-370.

8. Wasserman A, Brahn E (2010) Systemic sclerosis: bilateral improvement of Raynaud's phenomenon with unilateral digital sympathectomy. Semin Arthritis Rheum 40: 137-146.

9. Bier A (1908) Über einen neuen Weg, Lokalanästhesie an den Gliedmaßen zu erzeugen. Verh Dtsch Ges Chir 37: 204.

10. Murry CE, Jennings RB, Reimer KA (1986) Preconditioning with ischemia: a delay of lethal cell injury in ischemic myocardium. Circulation 74: 1124-1136.

11. Candilio L, Malik A, Hausenloy DJ (2013) Protection of organs other than the heart by remote ischemic conditioning. J Cardiovasc Med (Hagerstown) 14: 193-205.

12. Delagarde H, Ouadraougo N, Grall S, Macchi L, Roy PM, et al. (2015) Remote ischaemic preconditioning in intermittent claudication. Archives of Cardiovascular Disease 108: 472-479.

13. Puneet KR (2015) European Journal of Pharmacology 746: 317-332.

14. Heusch G, Bøtker HE, Przyklenk K, Redington A, Yellon D (2015) Remote Ischemic Conditioning. Journal of the American College of Cardiology.

15. Ferreira PCC (1989) Retrograde venous perfusion in the diabetic foot. In
Messmer K (eds): Ischemic diseases and the microcirculation. München, Zukschwerdt.

16. Agarwal P, Agrawal PK, Sharma D, Baghel KD (2005) Intervenous Infusion for the treatment of diabetic and ischemic non-healing pedal ulcers. J Eur Acad Dermatol Venereol 19:158-162.

17. Bühler-Singer S, Hiller D, Boateng B, Albrecht HP, Seidel C, et al. (1995) Disorderd cutaneous microcirculation in diabetic neuropathic foot ulcer. Is modification by retrograde transvenous perfusion therapy possible? Hautarzt 46: $400-405$.

18. Partsch H (1992) Treatment of Resistant Leg Ulcers by Retrograde Intravenous Pressure Infusions of Urokinase. Phlebology 7: 48-58.

19. Kommissari S (2002) Die retrograde venöse Perfusion. Therapieoption der diabetischen Gangrän und anderer infizierter Läsionen der Extremitäten. Prien, pp: $1-42$.

20. Langer K, Seidler C, Partsch H (1996) Ultrastructural Study of the Dermal Microvasculature in Patients Undergoing Retrograde Intravenous Pressure Infusions. Dermatology 192:103-109.

21. Kelm M (2002) Flow-mediated dilatation in human circulation: diagnostic and therapeutic aspects. Am J Physiol Heart Circ Physiol 282: H1-H5. 